J. Soc. Indust. Appl. Math.

Vol. 11, No. 1, March, 1963

Printed in U.S.A.

\title{
SINGULAR PERTURBATIONS OF BOUNDARY VALUE PROBLEMS INVOLVING ORDINARY DIFFERENTIAL EQUATIONS*
}

\section{A. ERDÉLYI $\dagger$}

1. Let us consider a boundary value problem, $P_{\epsilon}$, depending on a small parameter $\epsilon$ and assume that, as $\epsilon \rightarrow 0$, the differential equation and boundary conditions constituting $P_{\epsilon}$ approach limiting forms and define a "limiting boundary value problem", $P_{0}$. The solution, $y_{\epsilon}$, of $P_{\epsilon}$ depends on $\epsilon$, and under favourable conditions $y_{\epsilon}$ and an appropriate number of derivatives will approach limits, usually uniformly, as $\epsilon \rightarrow 0$; and $\lim y_{\mathrm{e}}$ will solve the limiting problem $P_{0}$. Moreover, in the event that $P_{\epsilon}$ depends on $\epsilon$ analytically, $y_{\epsilon}$ will also depend analytically on $\epsilon$ so that $y_{\epsilon}$ can be constructed by the usual perturbation calculations as a power series in $\epsilon$.

In this lecture we shall consider boundary value problems $P_{\epsilon}$ in which the order of the differential equation drops, or its type changes, as $\epsilon \rightarrow 0$ so that the boundary conditions prescribed in $P_{\epsilon}$ are not appropriate when $\epsilon=0$, and it is not at all obvious how $P_{0}$ should be defined. It is usually clear in such cases that $\lim y_{\epsilon}$, if it exists, cannot satisfy all the limiting boundary conditions. In many cases $\lim y_{\epsilon}$ will not be attained uniformly, indeed $\lim y_{\epsilon}$ may be a discontinuous function; and the derivatives of $y_{\epsilon}$ may fail to approach a limit or may be unbounded functions of $\epsilon$. A further characteristic feature of such "singular perturbation problems" is the nonanalytic dependence of $y_{\epsilon}$ on $\epsilon$ even in cases in which $P_{\epsilon}$ depends on $\epsilon$ in a very simple manner. This nonanalytic dependence expresses itself frequently in a markedly different behaviour as $\epsilon$ approaches zero through positive or negative values.

Singular perturbation problems occur in the theory of viscous flow, in certain problems in the theory of elasticity, and in many other branches of applied mathematics. Boundary layer theory furnishes some of the best known examples, and their study stimulated many of the more significant developments in this field. Clearly the most important problems of this nature involve partial differential equations. Nevertheless, we shall restrict ourselves in this lecture to singular perturbation problems involving ordinary differential equations and shall restrict the field further by assuming that the singular nature of the problem is caused entirely by a reduction of the order of the differential equation, and the consequent failure

* Received by the editors May 1, 1962. Invited address delivered at the meeting of SIAM in Pasadena, on March 24, 1962. The work was partly sponsored by the National Science Foundation under Grant No. G-19914.

$\dagger$ Department of Mathematics, California Institute of Technology, Pasadena, California. 
of some of the boundary conditions, as $\epsilon \rightarrow 0$. Additional complications (such as the emergence of singularities as $\epsilon \rightarrow 0$ ) will be excluded. Even in this narrowly restricted field we shall be unable to do more than outline briefly some of the known results and mention some of the unsolved problems.

2. A consideration of two very simple examples will show us some of the features of our singular perturbation problems.

The first example involves a linear differential equation with constant coefficients, and an explicit solution of the boundary value problem can be constructed. $P_{\epsilon}$ is given by

$$
\epsilon y^{\prime \prime}+y^{\prime}=0, \quad 0 \leqq t \leqq 1 ; \quad y(0)=y_{0}, \quad y(1)=y_{1} .
$$

The solution is

$$
y(t, \epsilon)=y_{0} \frac{e^{(1-t) / \epsilon}-1}{e^{1 / \epsilon}-1}+y_{1} \frac{1-e^{-t / \epsilon}}{1-e^{-1 / \epsilon}}
$$

Clearly $y$ fails to be analytic at $\epsilon=0$ and fails to approach a limit as $\epsilon \rightarrow 0$ unrestrictedly. One-sided limits exist, however, in half-open intervals.

$$
\begin{array}{lll}
y(t, \epsilon) \sim y_{0} e^{-t / \epsilon}+y_{1}, & 0<t \leqq 1, & \text { as } \quad \epsilon \rightarrow 0_{+}, \\
y(t, \epsilon) \sim y_{0}+y_{1} e^{(1-t) / \epsilon}, & 0 \leqq t<1, & \text { as } \quad \epsilon \rightarrow 0_{-},
\end{array}
$$

and hence

$$
\begin{aligned}
& y(t, \epsilon) \rightarrow y_{1} \text { in } 0<t \leqq 1 \text { as } \epsilon \rightarrow 0_{+}, \\
& y(t, \epsilon) \rightarrow y_{0} \text { in } 0 \leqq t<1 \text { as } \epsilon \rightarrow 0_{-} .
\end{aligned}
$$

Thus we have different limits according as $\epsilon$ approaches zero through positive or negative values. It is easy to verify that these limits are approached nonuniformly in their respective half-open intervals, and that the derivative, $y^{\prime}(t, \epsilon)$, is an unbounded function of $t$ and $\epsilon$. In both cases $\lim y$ satisfies the limiting differential equation, $y^{\prime}=0$, and it satisfies one, while in general it violates the other, boundary condition; which of the two boundary conditions is satisfied depends on the sign of $\epsilon$. Accordingly, $P_{0}$ is the boundary value problem $y^{\prime}=0,0 \leqq t \leqq 1, y(1)=y_{1}$ if $\epsilon \rightarrow 0_{+}$, while $P_{0}$ is given by $y^{\prime}=0,0 \leqq t \leqq 1, y(0)=y_{0}$ if $\epsilon \rightarrow 0_{-}$.

The second example is due to Coddington and Levinson [3] and shows that additional difficulties arise in the case of nonlinear differential equations. Here $P_{\epsilon}$ is given by

$$
\epsilon y^{\prime \prime}+y^{\prime}+y^{\prime 3}=0, \quad 0 \leqq t \leqq 1, \quad y(0)=y_{0},
$$$$
y(1)=y_{1} \text {. }
$$

The limiting differential equation is equivalent to $y^{\prime}=0$, and one might expect a behaviour somewhat resembling that encountered in connection 
with (2.1). Nevertheless, it will turn out that with the exception of the trivial case $y_{0}=y_{1}$ (when $\left.y(t, \epsilon)=y_{0}=y_{1}\right),(2.4)$ fails to possess any solution at all when $\epsilon$ is sufficiently small. It will be sufficient to consider the case $\epsilon \rightarrow 0_{+}$when the left end-point, $t=0$, might be expected to present difficulties. (If $\epsilon \rightarrow 0_{-}$, interchange the role of the two end-points.)

We know that $y(0)=y_{0}$. Let us set $y^{\prime}(0)=\tan \gamma,-\pi / 2<\gamma<\pi / 2$. Here $\gamma$ may depend on $\epsilon$ and $\tan \gamma$, as a function of $\epsilon$, may be unbounded. Now, the differential equation in (2.4) may be integrated explicitly in the form

$$
y(t, \epsilon)=y_{0}+\epsilon\left\{\gamma-\sin ^{-1}\left(e^{-t / \epsilon} \sin \gamma\right)\right\},
$$

where $\sin ^{-1}$ denotes the principal value, between $-\pi / 2$ and $\pi / 2$, of the inverse sine function. It follows that $\left|y(t, \epsilon)-y_{0}\right|<\epsilon|\gamma|$, and for given $y_{0}, y_{1}$ there will be no solution of $(2.4)$ when $0<\epsilon<2\left|y_{1}-y_{0}\right| / \pi$.

The nonexistence of the solution of (2.4) is generally attributed to the circumstance that the differential equation is nonlinear in $y^{\prime}$. For this reason some authors restrict themselves to differential equations that are linear in $y^{\prime}$ and $y^{\prime \prime}$ (but not necessarily in $y$ ). We shall see later that this restriction can be relaxed somewhat.

We now turn to a more general discussion of some aspects of singular perturbation problems and will in particular select the following: (i) The omission of boundary conditions, i.e., the formulation of $P_{0}$; (ii) the existence of a solution of $P_{\epsilon}$, and the relation of this solution to the solution of $P_{0}$; (iii) asymptotic expansions of the solution of $P_{\epsilon}$.

3. First we turn to the selection of those boundary conditions that must be discarded when constructing $P_{0}$. This problem has been studied in the case of linear differential equations by Wasow [12], Moser [8] for eigenvalue problems, and others. A recent exposition is due to B. Friedman [6].

Let us consider the differential equation

$$
(\epsilon N+M) y=0, \quad 0 \leqq t \leqq 1
$$

where

$$
\begin{aligned}
N y=\sum_{\nu=0}^{n} a_{\nu}(t) y^{(n-\nu)}, & M y=\sum_{\mu=0}^{m} b_{\mu}(t) y^{(m-\mu)}, & n>m, \\
a_{0}(t)=1, & b_{0}(t) \neq 0, & 0 \leqq t \leqq 1 ;
\end{aligned}
$$

together with $p$ boundary conditions, $A_{r}=0, r=1, \cdots, p$, at $t=0$ and $q$ boundary conditions, $B_{s}=0, s=1, \cdots, q$, at $t=1$. $A_{r}$ contains values of $y$ and its derivatives up to and including order $\alpha_{r}$ at $t=0, B_{s}$ belongs to $t=1$ and contains derivatives up to and including order $\beta_{s}$, and $p+q=n$. We assume that the boundary conditions have been replaced by equivalent boundary conditions such that no two $\alpha_{r}$ and no two $\beta_{s}$ are equal. 
The asymptotic behaviour of certain solutions of (3.1) as $\epsilon \rightarrow 0$ is known. Under appropriate conditions, (3.1) possesses $m$ solutions behaving asymptotically as $m$ linearly independent solutions of the limiting equation, $M y=0$, while further $n-m$ linearly independent solutions of (3.1) show an exponential dependence on $\epsilon$. The relevant exponential functions are the $n-m$ values of

$$
\exp \left\{\int\left[-\epsilon^{-1} b_{0}(t)\right]^{1 /(n-m)} d t\right\} .
$$

With two possible exceptions, corresponding to two possibly imaginary values of $\left[-\epsilon^{-1} b_{0}(t)\right]^{1 /(n-m)}$, the modulus of each of the functions (3.2) is either strictly increasing (as is $\exp (-t / \epsilon)$ in our first example in $\$ 2$ when $\epsilon<0$ ) or strictly decreasing (as is $\exp (-t / \epsilon)$ if $\epsilon>0$ ).

Corresponding to each increasing exponential function (3.2), Wasow omits a boundary condition at $t=1$ and corresponding to each decreasing function, a boundary condition at $t=0$. It is understood that boundary conditions of the highest order are omitted first, it is assumed that no two $A_{r}$ so omitted belong to values of $\alpha_{r}$ differing by a multiple of $n-m$, and a similar assumption is made with regard to the $\beta_{s}$. In the exceptional case, when two of the functions (3.2) are of modulus unity, Wasow omits the two remaining boundary conditions of the highest order, assuming that this can be done unambiguously. Thus, in all cases $n-m$ boundary conditions have been discarded; and the remaining $m$ boundary conditions together with the differential equation $M y=0$ define the limiting problem $P_{0}$.

Assuming further that $P_{0}$ possesses a unique solution $u(t)$, Wasow proves that for sufficiently small $\epsilon, P_{\epsilon}$ will also possess a unique solution $y(t, \epsilon)$, and $y(t, \epsilon) \rightarrow u(t)$ as $\epsilon \rightarrow 0$ provided $0<t<1$. The limit is approached uniformly in every closed subinterval of $(0,1)$, and the first $m$ derivatives of $y$ approach the corresponding derivatives of $u$. Finally, Wasow gives examples to show that in cases in which his rules cannot be followed or his assumptions are not satisfied, $y(t, \epsilon)$ need not approach a limit.

Wasow's differential equation (3.1) depends on $\epsilon$ in a very simple manner, and his boundary conditions are independent of $\epsilon$. An extension of these results to problems whose dependence on $\epsilon$ is more involved, and includes the dependence of the boundary conditions on $\epsilon$, was undertaken by Chapin [2] and Shabat [9]; and a further extension to systems of differential equations is due to Harris [7]. A somewhat different approach to the theory of a single $n$th order linear differential equation is found in the papers by Vishik and Lyusternik $[10,11]$ where there is also an extension to linear partial differential equations.

It should be possible to carry out similar investigations involving certain nonlinear differential equations, but no systematic investigations appear to 
be available in this direction, although corresponding investigations for the initial value problem have been made.

4. The second aspect we wish to investigate concerns the existence of a solution of $P_{\epsilon}$ and the relation of the solution of $P_{\epsilon}$ to that of $P_{0}$. In the case of linear equations the work described in $\$ 3$ provides much of the required information. Only the behaviour of $y$ near the end-points of the interval remains to be elucidated, and this can be accomplished by means of the asymptotic forms of the $n$ linearly independent particular solutions. For this reason we shall concentrate on nonlinear differential equations.

There is a considerable number of investigations involving nonlinear equations but there does not appear to be any very general or systematic theory. About the only more or less general class of nonlinear boundary value problems for which results are available is a class of problems $P_{\epsilon}$ involving a differential equation of the second order of the form

$$
\epsilon y^{\prime \prime}+F\left(t, y, y^{\prime}, \epsilon\right)=0, \quad 0 \leqq t \leqq 1
$$

and the boundary conditions

$$
y(0)=y_{0}, \quad y(1)=y_{1} .
$$

For the sake of simplicity we shall assume here that $y_{0}$ and $y_{1}$ are independent of $\epsilon$ although boundary conditions depending on $\epsilon$ could be included at the expense of some additional complications.

The limiting differential equation is of the first order and one of the boundary conditions must be discarded in forming $P_{0}$. We shall assume that $\epsilon \rightarrow 0_{+}$and presently will make assumptions on $F$ that will guarantee that the boundary condition at $t=0$ is the one to be discarded. Accordingly, let $P_{0}$ be the boundary value problem

$$
F\left(t, u, u^{\prime}, 0\right)=0, \quad 0 \leqq t \leqq 1, \quad u(1)=y_{1} .
$$

The basic assumption is that $P_{0}$ possesses a twice continuously differentiable solution $u$. The assumptions to be made on $F$ will ensure a smooth behaviour of $F$ when $y$ is near $u(t)$, and $y^{\prime}$ is near $u^{\prime}(t)$.

We expect that $y(t, \epsilon)-u(t) \rightarrow 0$ as $\epsilon \rightarrow 0_{+}$, nonuniformly in $0<t \leqq 1$, nonuniformity occurring at $t=0$. Let us borrow the terminology of boundary layer theory, call the immediate neighbourhood of $t=0$ the boundary layer, and call that part of the interval outside the boundary layer the outer region. We shall see that $y(t, \epsilon)-u(t)$ may be broken up, to some extent arbitrarily, in two parts. The first of these is the outer correction $v$ : it is uniformly small, indeed under the assumptions we are going to make it is $O(\epsilon)$; and it will turn out that $u+v$ is an outer approximation to $y$, valid up to an error that is exponentially small (in the outer region). The second part is the boundary layer correction, $w$; it is sizable in the boundary layer 
but exponentially small in the outer region. These two corrections will be constructed separately.

The problem $P_{\epsilon}$ given by (4.1) and (4.2) has been discussed under conditions of varying generality, and more or less detailed results have been obtained, by Coddington and Levinson [3], Briš [1], Wasow [13]. Here we shall follow Erdélyi [5] and assume that $P_{0}$ possesses a twice continuously differentiable solution $u$; that $F$ is defined and is twice continuously differentiable with respect to $y$ and $y^{\prime}$ in an appropriate region around $u$; that $F$ and its partial derivatives are continuous functions of $t, y, y^{\prime}$; that

$F\left(t, u(t), u^{\prime}(t), \epsilon\right)=O(\epsilon), \quad q(t, \epsilon)=F_{y}\left(t, u(t), u^{\prime}(t), \epsilon\right)=O(1)$,

$$
\phi(t)=\int_{0}^{t} F_{y^{\prime}}\left(\tau, u(\tau), u^{\prime}(\tau), 0\right) d \tau
$$

is twice continuously differentiable, $\phi^{\prime}(t)>0$,

$$
p(t, \epsilon)=F_{y^{\prime}}\left(t, u(t), u^{\prime}(t), \epsilon\right)=\phi^{\prime}(t)+\epsilon p_{1}(t, \epsilon),
$$

where $p_{1}(t, \epsilon)=O(1)$; and that $F_{y y}\left(t, y, y^{\prime}, \epsilon\right)=O(1), F_{y y^{\prime}}\left(t, y, y^{\prime}, \epsilon\right)$ $=O(1), F_{y^{\prime} y^{\prime}}\left(t, y, y^{\prime}, \epsilon\right)=O(\epsilon)$.

In these conditions and in the sequel subscripts $t, y, y^{\prime}$ denote partial differentiation and order relations, such as $O(\epsilon)$, are interpreted to hold as $\epsilon \rightarrow 0_{+}$, uniformly in all the other variables involved. The last condition, $F_{y^{\prime} y^{\prime}}=O(\epsilon)$ replaces the assumption of earlier writers to the effect that $F$ depend linearly on $y^{\prime}$.

To construct $v$, we shall prove that (4.1) possesses a solution $y^{*}$ satisfying the boundary conditions

$$
y^{* \prime}(0)=u^{\prime}(0), \quad y^{*}(1)=y_{1},
$$

and then set $y^{*}=u+v$. The boundary condition on $y^{*}$ at $t=0$ is arbitrary and corresponds to the arbitrariness in breaking up $y-u$ into $v$ and $w$.

$v$ satisfies a nonlinear differential equation that can be written in the form

$$
\epsilon v^{\prime \prime}+p v^{\prime}+q v=G\left(t, v, v^{\prime}, \epsilon\right),
$$

where $p$ and $q$ are defined in the assumptions, and $G$ is a certain combination of $F, F_{y}, F_{y^{\prime}}$ that we shall not display in detail. Suffice it to say that it can be deduced from our assumptions that

$$
\begin{aligned}
G(t, 0,0, \epsilon) & =O(\epsilon) \\
\left|G\left(t, z_{1}, z_{1}{ }^{\prime}, \epsilon\right)-G\left(t, z_{2}, z_{2}{ }^{\prime}, \epsilon\right)\right| & \leqq k \sigma\left(z_{2}, z_{1}{ }^{\prime}, z_{2}, z_{2}{ }^{\prime}\right)
\end{aligned}
$$

where $k$ is a constant and $\sigma$ is the largest of the two quantities

$$
\left|z_{1}-z_{2}\right| \max \left(\left|z_{1}\right|,\left|z_{2}\right|,\left|z_{1}^{\prime}\right|,\left|z_{2}{ }^{\prime}\right|\right)
$$


and

$$
\left|z_{1}^{\prime}-z_{2}^{\prime}\right| \max \left(\left|z_{1}\right|,\left|z_{2}\right|, \epsilon\left|z_{1}^{\prime}\right|, \epsilon\left|z_{2}^{\prime}\right|\right) \text {. }
$$

Let $V_{1}$ be that solution of the linear differential equation

$$
\epsilon V^{\prime \prime}+p V^{\prime}+q V=0
$$

satisfying the boundary conditions $V_{1}^{\prime}(0)=0, V_{1}(1)=1$; and let $K(t, s)$, $q u a$ function of $t$, be that solution of (4.8) satisfying, at $t=s$, the initial conditions $K(s, s)=0, \epsilon K_{t}(s, s)=1$. It follows from (4.6) and the boundary conditions $v^{\prime}(0)=0, v(1)=0$ that $v$ satisfies the nonlinear integro-differential equation

$$
\begin{aligned}
v(t)=\int_{0}^{t} K(t, s) G(s, v(s) & \left.v^{\prime}(s), \epsilon\right) d s \\
& -V_{1}(t) \int_{0}^{1} K(1, s) G\left(s, v(s), v^{\prime}(s), \epsilon\right) d s .
\end{aligned}
$$

The asymptotic behaviour of solutions of (4.8) can be investigated by known methods, and this investigation results in appraisals for $V_{1}$ and $K$. These appraisals may then be used in conjunction with the estimates (4.7) on $G$, to prove that, for sufficiently small $\epsilon$, a solution of (4.9) may be constructed by successive approximations, starting with $v_{0}(t)=0$. Moreover, this solution can be shown to satisfy

$$
v(t)=O(\epsilon), \quad v^{\prime}(t)=O(\epsilon) .
$$

$v$ is the outer correction, and $y^{*}=u+v$ is the outer solution.

Having constructed $v$, we can proceed to the construction of $w$, setting $y=y^{*}+w=u+v+w$ in (4.1), so that $w$ satisfies a nonlinear differential equation that can be written in the form

$$
\epsilon w^{\prime \prime}+p^{*} w^{\prime}+q^{*} w=G^{*}\left(t, w, w^{\prime}, \epsilon\right),
$$

where

$$
p^{*}=F_{y^{\prime}}\left(t, y^{*}(t), y^{* \prime}(t), \epsilon\right), \quad q^{*}=F_{y}\left(t, y^{*}(t), y^{* \prime}(t), \epsilon\right),
$$

and $G^{*}$ is given in terms of $F, F_{y}, F_{y^{\prime}}$ and is such that under our conditions

$$
\begin{aligned}
G^{*}(t, 0,0, \epsilon) & =0 \\
\left|G^{*}\left(t, z_{1}, z_{1}{ }^{\prime}, \epsilon\right)-G^{*}\left(t, z_{2}, z_{2}{ }^{\prime}, \epsilon\right)\right| & \leqq k^{*} \sigma\left(z_{1}, z_{1}{ }^{\prime}, z_{2}, z_{2}{ }^{\prime}\right) .
\end{aligned}
$$

In addition to (4.11), $w$ also satisfies the boundary conditions

$$
w(0)=y_{0}-u(0)-v(0), \quad w(1)=0 .
$$

We are now in a situation similar to that encountered in connection with 
$v$. We can show that $w$ satisfies a nonlinear integro-differential equation and may attempt a construction of $w$ by successive approximations, starting with $w_{0}(t)=0$. The situation is different in one respect, though. (4.12) shows that $w(t)$ is not uniformly small, and the convergence of the successive approximations for small $\epsilon$ will not be automatic. Nevertheless, asymptotic appraisals of certain solutions of the linear differential equation

$$
\epsilon W^{\prime \prime}+p^{*} W^{\prime}+q^{*} W=0
$$

in conjunction with the above estimates on $G^{*}$ may be used to establish the existence of a positive number $\mu_{0}$ independent of $\epsilon$ and such that whenever $\left|y_{0}-u(0)\right|<\mu_{0}$ and $\epsilon$ is sufficiently small, the successive approximations converge to a solution $w$ of (4.11), (4.12). Moreover,

$$
w(t)=O\left(e^{-\phi(t) / \epsilon}\right), \quad \epsilon w^{\prime}(t)=O\left(e^{-\phi(t) / \epsilon}\right)
$$

for this solution. This last estimate shows that $w$ is indeed a boundary layer correction that vanishes exponentially in the outer region. $y=u+v+w$ is an exact solution of $P_{\epsilon}$, while $u$ is a solution of $P_{0}$. The process outlined above establishes the existence of $y$ for sufficiently small $\epsilon$, and shows that

$$
\begin{gathered}
y(t, \epsilon)=u(t)+O(\epsilon)+O\left(e^{-\phi(t) / \epsilon}\right), \\
y^{\prime}(t, \epsilon)=u^{\prime}(t)+O(\epsilon)+O\left(\frac{1}{\epsilon} e^{-\phi(t) / \epsilon}\right) .
\end{gathered}
$$

5. The third aspect we wish to discuss is the existence, under conditions more restrictive than those imposed above, of asymptotic expansions for $\epsilon \rightarrow 0_{+}$. The nature of the order terms in (4.14), together with examples showing that these order terms are realistic, indicates that for $0 \leqq t \leqq 1$, $y(t, \epsilon)$ cannot possess asymptotic expansions in powers of $\epsilon$ with coefficients depending on $t$ but independent of $\epsilon$. At the very least, asymptotic expansions valid for $0 \leqq t \leqq 1$ will involve exponential functions of $\epsilon$ as well as powers.

In the case of a linear boundary value problem, such as the one discussed in $\S 3$, the known asymptotic expansions of particular solutions of the differential equation will lead to an asymptotic expansion of the solution of $P_{\epsilon}$. Nonlinear boundary value problems of the form (4.1) were investigated by Wasow [13] under the assumption that $F$ is linear in $y^{\prime}$ and analytic in $t, y$, and $\epsilon$. Wasow proved that under these assumptions $y(t, \epsilon)$ possesses a convergent expansion in powers of $\exp [-\phi(t) / \epsilon]$ whose coefficients are functions of $t$ and $\epsilon$; and that these coefficients possess asymptotic expansions in powers of $\epsilon$.

Expansion techniques applicable to a wide variety of problems have been developed in connection with boundary layer theory. We shall describe 
briefly the technique developed by Lagerstrom, Cole, Kaplun, and their associates (see references in [4]); and we note in passing that similar expansions have been used also by Proudman, Mahoney, Pillow, and others. For the sake of simplicity, we shall describe the application of the technique in question to the problem $P_{\epsilon}$ of $\S 4$ although this technique has been applied successfully to other types of problems, including boundary value problems involving partial differential equations.

Let $y(t, \epsilon)$ be the solution of $P_{\epsilon}$. Under appropriate assumptions on $F, y$ will possess an asymptotic expansion as $\epsilon \rightarrow 0_{+}$while $t>0$ is fixed; and in many cases this asymptotic expansion will be a power series in $\epsilon$ with coefficients depending on $t$. This expansion will be called the outer expansion and will be denoted by $y^{0}$. From its derivation, one would expect the outer expansion to hold on any interval $\delta \leqq t \leqq 1$ with $\delta>0$ independent of $\epsilon$, but actually the situation is more favourable. Equation (4.13) suggests that the outer expansion may hold for $\delta \leqq t \leqq 1$ even if $\delta$ depends on $\epsilon$ and approaches zero as $\epsilon \rightarrow 0_{+}$, provided only that $\delta / \epsilon \rightarrow \infty$; this can be verified under certain assumptions on $F$.

In order to obtain an asymptotic expansion valid in the boundary layer, we introduce a stretching transformation, $t=\tau \epsilon($ or $\phi(t)=\tau \epsilon)$. The asymptotic expansion of $y(\tau \epsilon, \epsilon)$ for $\epsilon \rightarrow 0_{+}$while $\tau \geqq 0$ is fixed is called the inner expansion and is denoted by $y^{i}$. This expansion will be expected to hold when $0 \leqq \tau \leqq A$ for some $A$, i.e., if $t=O(\epsilon)$, but examples show that in fact it holds in a larger region, in some cases when $t=o(1)$. Thus, both the inner and the outer expansions hold in an overlap region, in the circumstances outlined here, when both $t=o(1)$ and $\epsilon / t=o(1)$.

The overlap region can be used to match the outer and inner expansions. In that region it makes sense to form the outer expansion of the inner expansion, $\left(y^{i}\right)^{0}$, and vice versa, $\left(y^{0}\right)^{i}$. The Lagerstrom-Kaplun matching principle states that the two expansions so obtained are related to each other by the stretching transformation. Once the two expansions are matched, a composite expansion can be formed according to the rule $y^{c}=y^{0}+y^{i}-\left(y^{0}\right)^{i}=y^{0}+y^{i}-\left(y^{i}\right)^{0} ;$ and under appropriate conditions this composite expansion provides a uniform approximation throughout the interval $0 \leqq t \leqq 1$.

Let us indicate briefly the application of this technique to the boundary value problem $P_{\epsilon}$ of $\S 4$. The resulting asymptotic expansions will not necessarily proceed in integral powers of $\epsilon$; indeed the determination of the "asymptotic scale", of the sequence of functions appearing in the asymptotic expansions, may present a formidable practical difficulty and may have to be carried out step by step. Nevertheless, we shall assume for the sake of simplicity that $P_{\epsilon}$ is of such a nature as to lead to asymptotic expansions in integral powers of $\epsilon$. 
The outer expansion will be of the form

$$
y^{0}=f_{0}(t)+\epsilon f_{1}(t)+\epsilon^{2} f_{2}(t)+\cdots .
$$

Clearly, $f_{0}(t)=u(t)$ is the solution of $P_{0}$, and substitution of (5.1) in (4.1) and comparison of like powers of $\epsilon$ leads to recurrent differential equations of the first order for the $f_{n}$, so that the boundary condition at $t=1$ determines the $f_{n}$ uniquely.

With the stretching transformation $t=\epsilon \tau$, the inner expansion will be of the form

$$
y^{i}=g_{0}(\tau)+\epsilon g_{1}(\tau)+\cdots,
$$

while the differential equation (4.1) now appears in the form

$$
\ddot{y}+\epsilon F\left(\epsilon \tau, y, \frac{1}{\epsilon} \dot{y}, \epsilon\right)=0,
$$

where

$$
\dot{y}=\frac{d y}{d \tau}=\epsilon y^{\prime}, \quad \ddot{y}=\frac{d^{2} y}{d \tau^{2}}=\epsilon^{2} y^{\prime \prime} .
$$

One of the conditions for the feasibility of an inner expansion is the existence of the limit

$$
F_{0}(\tau, y, \dot{y})=\lim _{\epsilon \rightarrow 0+} \epsilon F\left(\epsilon \tau, y, \frac{1}{\epsilon} \dot{y}, \epsilon\right) .
$$

Assuming this, $g_{0}$ will satisfy the differential equation

$$
\ddot{g}_{0}+F_{0}\left(\tau, g_{0}, \dot{g}_{0}\right)=0 .
$$

We have only one boundary condition for $g_{0}$, namely a boundary condition at $\tau=0$, so that $g_{0}$, being a solution of a differential equation of the second order, will contain an undetermined constant. Similarly, each $g_{n}$ will contain an undetermined constant.

The matching principle will now be used for the determination of the constants in the $g_{n}$. Using the overlap region for matching, $f_{n}(\epsilon \tau)$ may be expanded in powers of $\epsilon$, and $g_{n}(t / \epsilon)$ may also be expanded in powers of $\epsilon$. The two expansions so obtained, $\left(y^{0}\right)^{i}$ and $\left(y^{i}\right)^{0}$, when related to each other by means of the stretching transformation, determine the unknown constants. Once this is accomplished, the composite expansion may be constructed.

We shall illustrate this process by obtaining the composite approximation of order zero for $P_{\epsilon}$ in the special case when

$$
F\left(t, y, y^{\prime}, \epsilon\right)=F_{1}(t, y, \epsilon) y^{\prime}+F_{2}(t, y, \epsilon) .
$$


From the work of $\S 4$,

$$
y^{0}=u(t)+\cdots
$$

in this case, and

$$
\left(y^{0}\right)^{i}=u(0)+\cdots,
$$

since $u(\epsilon \tau)=u(0)+O(\epsilon \tau)$. We have

$$
F_{0}(\tau, y, \dot{y})=\lim _{\epsilon \rightarrow 0+} F_{1}(\epsilon \tau, y, \epsilon) \dot{y},
$$

and $g_{0}$ is a solution of $(5.3)$. If $g_{0}(t / \epsilon)$ is to possess an asymptotic expansion in the overlap region, where $t / \epsilon \rightarrow \infty$, then clearly this expansion must begin with $g_{0}(\infty)$, so that

$$
y^{i}=g_{0}(\tau)+\cdots, \quad\left(y^{i}\right)^{0}=g_{0}(\infty)+\cdots .
$$

The matching principle, relating $\left(y^{0}\right)^{i}$ and $\left(y^{i}\right)^{0}$, demands $u(0)=g_{0}(\infty)$, so that $g_{0}$ is now determined as that solution of the differential equation (5.3) satisfying the boundary conditions $g_{0}(0)=y_{0}, g_{0}(\infty)=u(0)$. Finally, the composite expansion will be

$$
y^{c}=u(t)+g_{0}(t / \epsilon)-u(0)+\cdots .
$$

In order to establish the validity of this technique, one would have to show that

$$
y(t, \epsilon)-u(t)-g_{0}(t / \epsilon)+u(0) \rightarrow 0,
$$

uniformly for $0 \leqq t \leqq 1$, as $\epsilon \rightarrow 0_{+}$. Writing $y=y^{*}+w$, we know from $\$ 4$ that

$$
v(t, \epsilon)=y^{*}(t, \epsilon)-u(t)=O(\epsilon) \quad \text { as } \quad \epsilon \rightarrow 0_{+} .
$$

There remains to be shown that

$$
w(t, \epsilon)-g_{0}(t / \epsilon)+u(0) \rightarrow 0 \text { as } \epsilon \rightarrow 0_{+} .
$$

This relation has been verified in certain examples, but a general proof has not as yet been accomplished. Work in this direction continues.

In the overlap region neither $t$ nor $\tau$ can be "fixed". In the outer region we assume that $t$ is bounded away from zero, in the inner region $t=O(\epsilon)$, while in the overlap region we have an intermediate situation in which $t$ approaches zero but not too rapidly; and the limiting processes involved in a satisfactory formulation of the matching principle refer to this intermediate situation. Lagerstrom and Kaplun developed a comprehensive theory of intermediate limits that goes a long way towards giving an insight in the whole process and making the matching principle plausible. At the 
present stage of its development, the theory of intermediate limits cannot as yet prove the matching principle or delineate its region of validity. Nevertheless, the technique described in the present section has been used in numerous problems in applied mathematics and yielded very satisfactory results. There are also simple examples of boundary value problems for which the matching principle and the uniform validity of the composite expansion can be demonstrated mathematically, either by reference to a known exact solution or, in cases when the exact solution is not known, by integral equation methods.

\section{REFERENCES}

[1] N. I. BRǏ̌, On boundary problems for the equation $\epsilon y^{\prime \prime}=f\left(x, y, y^{\prime}, \epsilon\right)$ for small $\epsilon$ 's, Dokl. Akad. Nauk SSSR, 95 (1954), pp. 429-432.

[2] G. G. Chapin Jr., One and two point boundary value problems for ordinary linear differential equations containing a parameter, Tech. Rep. No. 4, OOR Project No. 1469, (1959), University of Minnesota.

[3] E. A. Codpington and N. Levinson, A boundary value problem for a nonlinear differential equation with a small parameter, Proc. Amer. Math. Soc., 3 (1952), pp. 73-81.

[4] A. ERDÉlyi, An expansion procedure for singular perturbations, Atti Accad. Sci. Torino. Cl. Sci. Fis. Mat. Nat., 95 (1960-61), pp. 651-672.

[5] - Singular perturbations, Bull. Amer. Math. Soc., 68 (1962), pp. 420-424.

[6] Bernard Friedman, Singular perturbations of ordinary differential equations, Math. Note No. 245, Boeing Scientific Research Laboratories, (1961).

[7] W. A. HARris JR., Singular perturbations of two-point boundary value problems for systems of ordinary differential equations, Arch. Rational Mech. Anal., 5 (1960), pp. 212-225.

[8] JÜRGEN MOSER, Singular perturbation of eigenvalue problems for linear differential equations of even order, Comm. Pure Appl. Math., 8 (1955), pp. 251278.

[9] A. B. Shabat, Boundary value problems with small parameters for linear ordinary differential equations, Uspehi Mat. Nauk., 17 (1962), pp. 235-241.

[10] M. I. Vishik AND L. A. Lyusternik, Regular degeneracy and boundary layer for linear differential equations with a small parameter, Ibid., 12 (1957), pp. 3-122.

[11] - Solution of some perturbation problems in the case of matrices and selfadjoint or nonselfadjoint equations, Russian Math. Surveys, 15 (1960), pp. $1-73$.

[12] W. WAsow, On the asymptotic solution of boundary value problems containing a parameter, J. Math. Phys., 23 (1944), pp. 175-183.

[13] - - Singular perturbations of boundary value problems for nonlinear differential equations of the second order, Comm. Pure Appl. Math., 9 (1956), pp. 93-113. 